\title{
Effect Analysis of Intermittent Release Measures in Heavy Fog Weather with an Improved CA Model
}

\author{
Jing Shi and Jinhua Tan \\ Institute of Transportation Engineering, Tsinghua University, Beijing 100084, China \\ Correspondence should be addressed to Jing Shi; jingshi@mail.tsinghua.edu.cn
}

Received 3 August 2013; Accepted 10 October 2013

Academic Editor: Tetsuji Tokihiro

Copyright ( 2013 J. Shi and J. Tan. This is an open access article distributed under the Creative Commons Attribution License, which permits unrestricted use, distribution, and reproduction in any medium, provided the original work is properly cited.

\begin{abstract}
Heavy fog may easily cause traffic accidents; thus freeway closures are frequently taken in order to ensure traffic safety in China, which not only seriously affect the travel of people, but also bring great economic losses. This paper studies the fog related risk of rear-end collisions and the intermittent release measures taken to reduce such risk; meanwhile, an improved cellular automaton model considering driving behaviors in heavy fog is proposed. The simulation results indicate that the risk indicator $f_{a}$ in fog is much higher than normal weather when cellular occupancy $\rho<0.5$. After taking intermittent release measures, the magnitude of $f_{a}$ will drop from $10^{-4}$ to $10^{-5}$ under the same fog condition, which greatly enhances the safety. In addition, this paper concludes the appropriate vehicle number released for each time and the time interval $h_{t}$ between adjacent fleets and the maximum number of vehicles $Q_{\max }$ which can be released per hour. These results can be used as theoretical basis and reference for the traffic management departments to develop intermittent release measures.
\end{abstract}

\section{Introduction}

Traffic accidents happen frequently in heavy fog weathers. According to the U.S. Federal Highway Administration, there are about 600 people killed and 16, 300 injured annually in fog related road traffic accidents in the United States [1]. On the Shanghai-Nanjing freeway in China, the heavy fog related traffic accidents have accounted for a quarter of the total accidents, and the accident rate during heavy fog weather was 10 times over those in normal weather [2].

The freeway will be temporarily closed if the visibility drops down to 50 meters according to the Announcement of the Freeway Traffic Management in Low Visibility Weather Conditions issued by the Ministry of Public Security of the People's Republic of China [3]. By the end of 2012, the freeway mileage in China amounted to 96, 200 kilometers. For traffic safety reasons, freeways are frequently closed in heavy fog, which not only seriously affects travelers' mobility, but also brings a great deal of economic losses. Take ShanghaiNanjing freeway as an example, the mileage is $274.35 \mathrm{~km}$, and there were 57 closures due to heavy fog from 2006 to 2009. The direct economic losses caused by closures are over RMB67.81 million Yuan [2]. Therefore, the heavy fog has become the main adverse weather which threatens the normal operation of freeways.

To reduce losses and take into account the traffic safety, some areas start to use intermittent release measures instead of road closures in heavy fog weather. Typical intermittent release measure is that traffic management department releases a certain number of passenger cars with a certain period of time interval. Generally, large trucks and dangerous goods transport vehicles are not allowed to be released. And some driving behaviors are prohibited, such as speeding, overtaking, and lane changing. However, there exist risks when the intermittent release measure is enforced. Previous studies show that low visibility and specific driving behaviors in heavy fog are the two key risk factors that impact on the measures [4-7].

The intermittent release measures can be simulated by using cellular automaton (CA) model of traffic. Cellular automaton is a dynamic system, which is composed of discrete, finite state cells, in accordance with the certain local rules. Wolfram's 184 rule can be used to describe the movement of vehicles [8]. As the promotion of rule 184, Nagel and Schreckenberg advanced one-dimensional 


\begin{tabular}{|c|c|}
\hline $\mid \longleftarrow$ & Visibility \\
\hline & $\longrightarrow$ \\
\hline
\end{tabular}

(a)

\begin{tabular}{l|l|l|l|l|l|l|l|}
\hline \hline & $\vdots$ & $\vdots$ & $\vdots$ & $\vdots$ & $\rightarrow$ & $\vdots$ & \\
\hline
\end{tabular}

(b)

FIgURE 1: Diagram of the CA model in heavy fog. Note: the single arrows in the figure show the driving direction.

cellular automaton model [9]. Since then, a large number of scholars put forward new improved models, such as FI model and comfortable driving model $[10,11]$. Boccara et al. have developed the road traffic safety judgment method by using the CA model [12]. Later, corresponding follow-up studies have been made $[13,14]$, such as Moussa; a conclusion was drawn that the probability of traffic accidents is higher when the space occupancy of vehicle is lower. Lárraga et al. defined a safety parameter in the reduction step of $\mathrm{NaSch}$ model rules, to reflect the traffic safety [15]. In recent years, scholars have been more concerned about the driving characteristics and driving behaviors. Wu et al. divided the drivers into different types in order to study the impact of different driving characteristics on the traffic $[16,17]$. Some certain driving behaviors which influence traffic flow have also been studied $[18,19]$.

This paper proposes an improved cellular automaton model of traffic that considers driving behaviors in heavy fog, in order to study the freeway intermittent release measures and reduce the fog related traffic risk. Firstly, the impacts of heavy fog on traffic speed and capacity are discussed. Secondly, the probability of traffic accidents per vehicle per second on average is studied. Finally, a reasonable release number and time interval of the fleets are determined.

\section{Driving Behaviors in Heavy Fog and CA Model}

Affected by low visibility, the driving behaviors in heavy fog differ greatly from those in normal weather. Some scholars studied the driving behaviors in heavy fog, and the results are mainly manifested in the following two points.

(1) A few drivers may become laggers in queue as the space headway is greater than the visibility in heavy fog [4], as shown in Figure 1(a). The randomization deceleration degree of laggers is greater than others when all the vehicles have the same randomization deceleration probability, if it is explained within the framework of the NaSch model.

(2) The drivers will feel risky when driving in fog, leading to the corresponding changes in driving behaviors such as speeding up unintentionally for shortening the space headway $[5,6]$. Duan explained that the vehicles will only appear in closer distance range in heavy fog, as shown in Figure 1(b). And some drivers may unconsciously accelerate to shorten the following distance in order to increase the stability of the vision and to gain more sense of safety [7]. Obviously, this behavior may increase the risk of rear-end collision. Similarly, as it is explained within the framework of the NaSch model, the randomization deceleration degree is relatively small when all the vehicles have the same randomization deceleration probability.

In order to describe the characteristics of the driving behaviors in heavy fog, this paper introduces the concept of the degree of randomization deceleration on the basis of the NaSch model and proposes an improved cellular automaton model of traffic.

In the model, fog zone length is set as $L$ cells, and each cell represents $L_{\text {cell }}$ meters. The first vehicle is marked as $N$, and the following are in turn marked as $N-1, N-2, \ldots, 1$. The location of the $n$th vehicle at time $t$ is marked as $x_{n}(t)$, and the speed is marked as $v_{n}(t)$. The time step is taken to be 1 second. Let $v_{\max }$ denote the maximum velocity. Let $d_{v}$ and $d_{\text {safe }}$ denote the visibility and preselected threshold, and the drivers are not affected by heavy fog when the space headway is less than $d_{\text {safe }}$. Let $a_{1}, a_{2}$ and $a_{3}$ denote the degree of randomization deceleration depending on space headway at a given randomization deceleration probability $P$. Obviously, the randomization deceleration rule of the NaSch model is recovered when $a_{1}=a_{2}=a_{3}=1$, which describes the traffic operating conditions in normal weather. The model assumes that only passenger cars are contained and no overtaking behaviors in the fleet. Meanwhile, it is also regulated that vehicles must strictly follow the speeds controlled by the leading vehicle. State transitions are defined with the following set of rules, which are applied simultaneously to all vehicles:

(1) acceleration:

$$
v_{n}\left(t+\frac{1}{3}\right)=\min \left(v_{n}(t)+1, v_{\max }\right)
$$

(2) slowing down:

$$
v_{n}\left(t+\frac{2}{3}\right)=\min \left(v_{n}\left(t+\frac{1}{3}\right), x_{n+1}(t)-x_{n}(t)-1\right),
$$




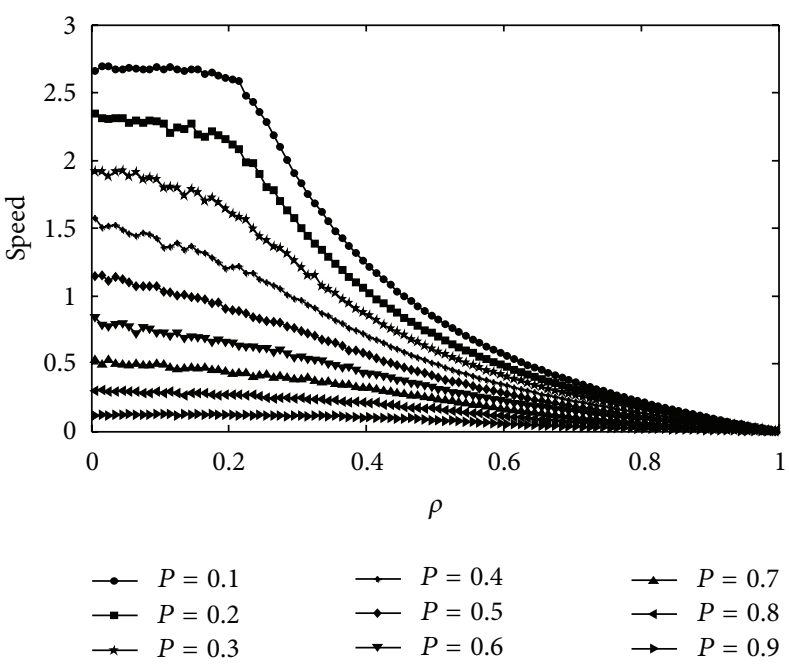

(a)

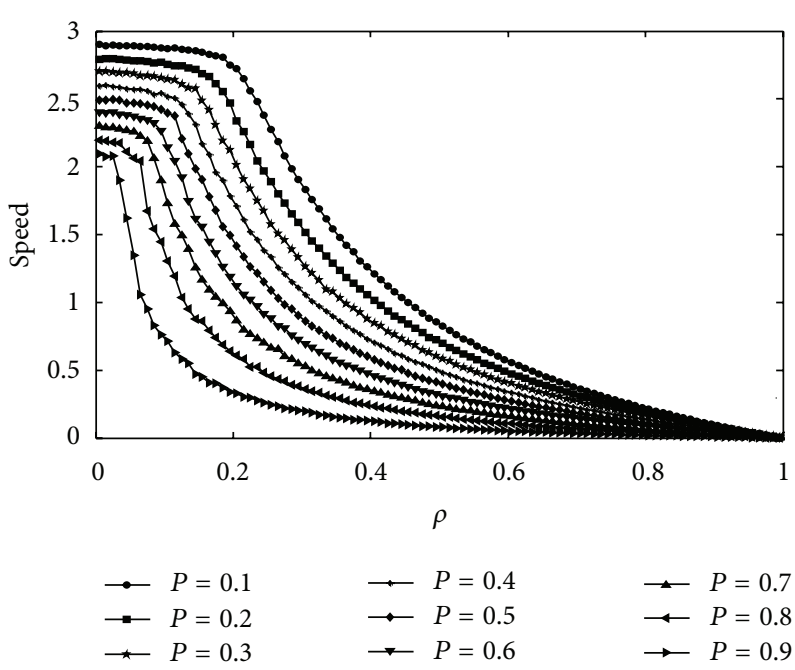

(b)

FIGURE 2: Relationship between traffic speed and $\rho$ : (a) heavy fog condition and (b) normal weather condition.

(3) randomization deceleration:

$$
\begin{aligned}
& v_{n}(t+1) \\
& = \begin{cases}\max \left(v_{n}\left(t+\frac{2}{3}\right)-a_{1}, 0\right) & \text { for } x_{n+1}(t)-x_{n}(t) \\
& >d_{v} \\
\max \left(v_{n}\left(t+\frac{2}{3}\right)-a_{2}, 0\right) & \text { for } d_{\text {safe }}<x_{n+1}(t) \\
& -x_{n}(t) \leq d_{v} \\
\max \left(v_{n}\left(t+\frac{2}{3}\right)-a_{3}, 0\right) & \text { for } x_{n+1}(t)-x_{n}(t) \\
& \leq d_{\text {safe }},\end{cases}
\end{aligned}
$$

(4) vehicle motion:

$$
x_{n}(t+1)=x_{n}(t)+v_{n}(t+1) \text {. }
$$

\section{Model Parameters of Intermittent Release Measures}

The model proposed here is a probabilistic cellular automaton. It consists of $N$ vehicles moving in a one-dimensional lattice of $L$ cells under periodic boundary conditions, each cell may either be empty or be occupied by one vehicle; the parameter values are $L=1000, L_{\text {cell }}=6, v_{\max }=3, d_{v}=$ $8, a_{1}=2, a_{2}=0, a_{3}=1, d_{\text {safe }}=6, \rho=N / L$, and $\rho_{\text {min }}=0.005$. The cellular occupancy $\rho$ here is equivalent to the traffic density. Different values of $N$ and $P$ were simulated. The simulation was repeated 20 times for getting the average value in order to eliminate the influence of random factors as much as possible.
TABLE 1: Traffic capacity with the corresponding values of $\rho$.

\begin{tabular}{lccc}
\hline$P$ & 0.1 & 0.4 & 0.6 \\
\hline Normal weather & & & \\
$\quad \rho$ & 0.26 & 0.18 & 0.16 \\
$\quad$ Traffic capacity & 0.58 & 0.35 & 0.24 \\
Heavy fog weather & & & \\
$\quad \rho$ & 0.26 & 0.32 & 0.34 \\
$\quad$ Traffic capacity & 0.58 & 0.29 & 0.18 \\
\hline
\end{tabular}

3.1. The Heavy Fog Related Traffic Characteristics. First of all, the impact of heavy fog on the traffic reflects in the reduction of speed.

Figure 2 shows the contrast of speed in heavy fog and normal weather conditions with the variation of cellular occupancy $\rho$. The maximum speed is 2.68 in heavy fog, but 2.89 in normal weather when $P=0.1$. However, with the increasing of $\rho$, the decrease of speed in heavy fog is smaller than that in normal weather. Take $\rho=0.5$ for example, the maximum speed is 0.82 in heavy fog and 0.83 in normal weather. In heavy fog, the impact of $P$ on speed is greater than that in normal weather. Take $P=0.4$ for example, the maximum speed is just 1.52 in heavy fog, but 2.60 in normal weather.

The impact of heavy fog on traffic capacity is also very obvious. It is illustrated in Figure 3 that with the increasing of $P$, the corresponding values of $\rho$ in heavy fog are bigger than in normal weather when the maximum volume appears, as shown in Table 1 . The heavy fog has no significant impact on the traffic capacity when $P$ is too small or too large, as shown in Table 2.

3.2. Parameters Determination. The simulation parameters determination of the intermittent release measures mainly include randomization deceleration probability $P$ and 


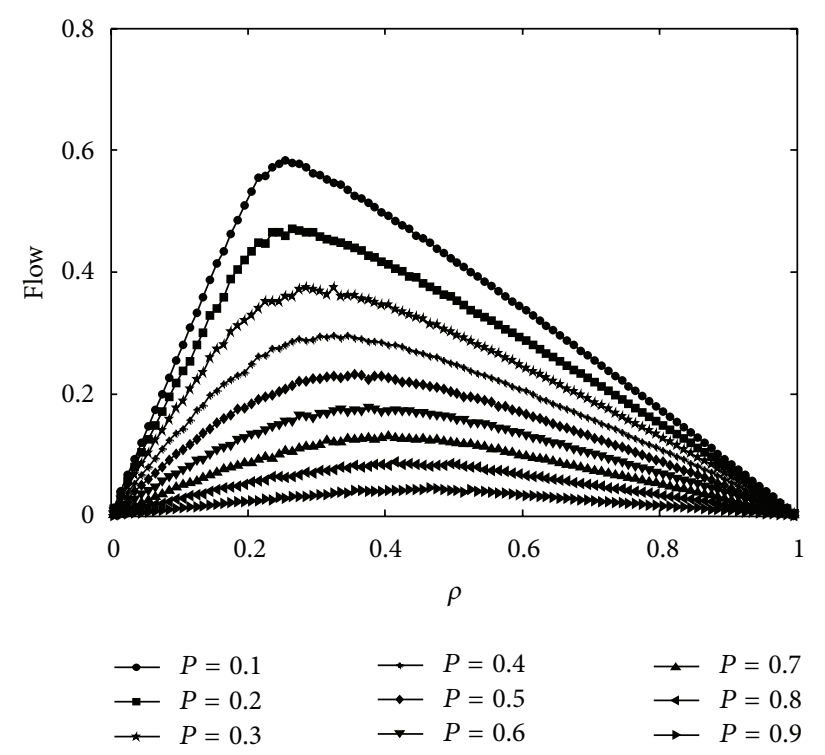

(a)

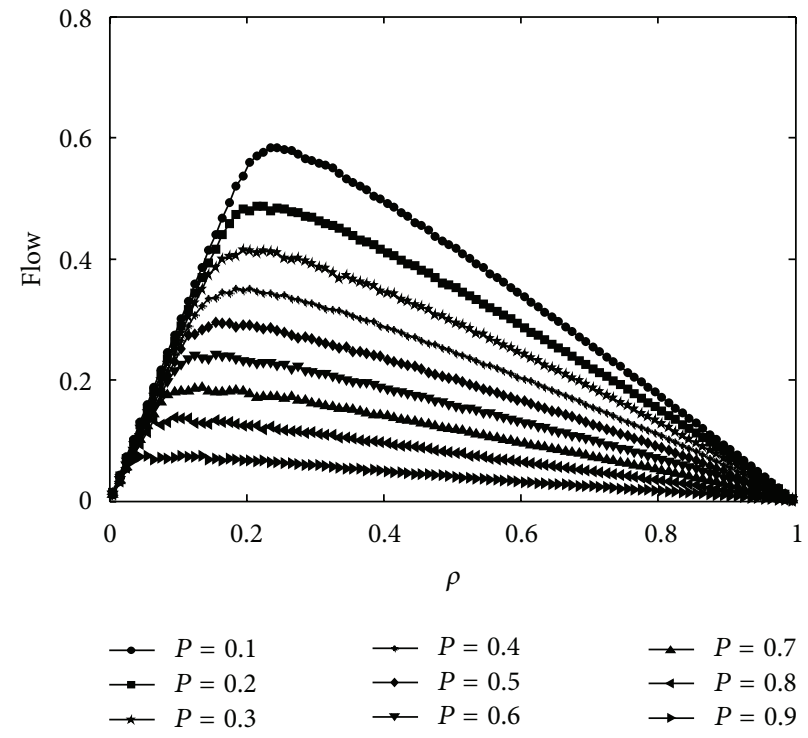

(b)

FIGURE 3: Relationship between traffic capacity and $\rho$ : (a) heavy fog condition and (b) normal weather condition.

TABLE 2: The relative difference of traffic capacity in heavy fog and normal weather.

\begin{tabular}{lccccccccc}
\hline$P$ & 0.1 & 0.2 & 0.3 & 0.4 & 0.5 & 0.6 & 0.7 & 0.8 & 0.9 \\
\hline Normal weather & 0.58 & 0.49 & 0.41 & 0.35 & 0.29 & 0.24 & 0.19 & 0.14 & 0.07 \\
Heavy fog weather & 0.58 & 0.47 & 0.38 & 0.29 & 0.23 & 0.18 & 0.13 & 0.09 & 0.05 \\
Relative difference & 0 & 0.02 & 0.03 & 0.06 & 0.06 & 0.06 & 0.06 & 0.05 & 0.02 \\
\hline
\end{tabular}

the proportion of the drivers suffering from accidents caused by distraction $f_{d}$ in this paper.

According to HCM2010, the traffic capacity in heavy fog is about $11 \%$ lower than that in normal weather [20]. The simulation experiment results of this paper show that the traffic capacity is about $11 \%$ lower when $P=0.31$. We investigate the problem of traffic safety in heavy fog conditions assuming $P=0.31$.

Boccara et al. proposed three conditions that can be used for measuring the frequency of dangerous situations (DS) and pointed out that the accident may occur if the driver diverts his attention in the DS [12]. The proportion of the drivers suffering from accidents caused by distraction is recorded as $f_{d}$. A survey about driving behaviors was conducted in 2011. The results showed that about $1.9 \%$ drivers always have distracted driving behaviors [21]. Therefore, $f_{d}=1.9 \%$ is used in this paper. The probability of traffic accidents per vehicle per second on average can be represented by $f_{a}$, as shown in (5). The curve of $f_{a}$ in heavy fog and normal conditions is shown in Figure 4. Consider

$$
f_{a}=f_{d} \frac{1}{N} \frac{1}{T} \sum_{t=1}^{T} \sum_{n=1}^{N} \mathrm{DS}(t) .
$$

Figure 4 shows that the value $f_{a}$ in heavy fog is greater than in normal weather when $\rho<0.65$, and the lower $\rho$ is, the greater relative difference is. Therefore, driving in heavy

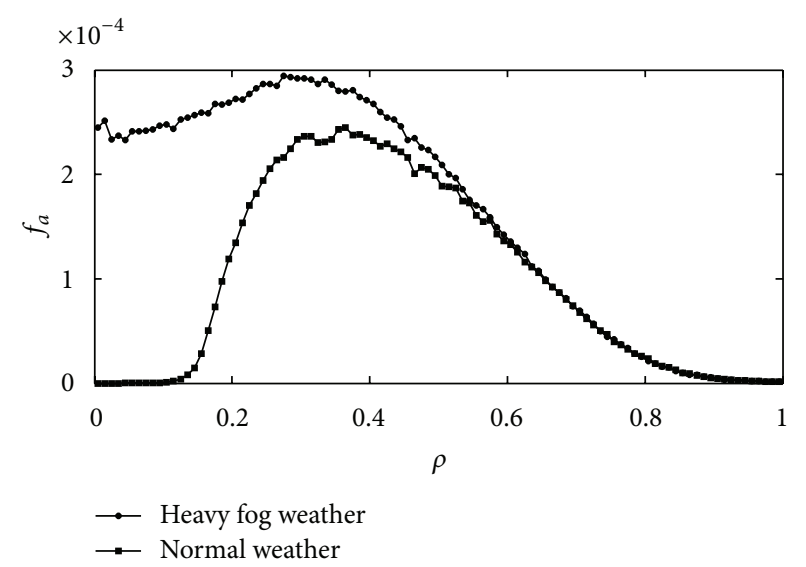

FIGURE 4: Relationship between $f_{a}$ and $\rho$.

fog is risky due to the impact of the visibility and the specific driving behaviors. Thus, traffic control measures should be taken to ensure traffic safety.

\section{Simulation Results and Analysis of Intermittent Release Measures}

Freeway intermittent release measures mainly include the vehicle number $N$ of each fleet and the time interval $h_{t}$ 


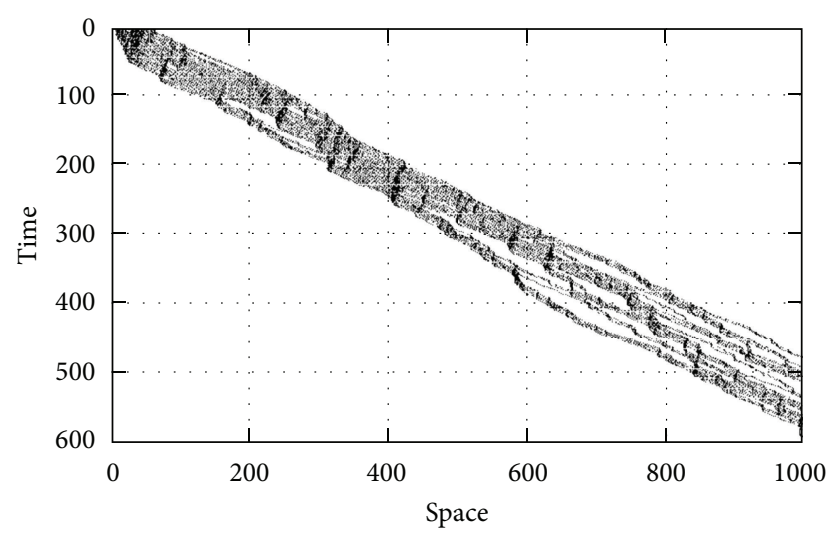

FIGURE 5: The space-time diagram obtained from the model with $N=30, P=0.31$

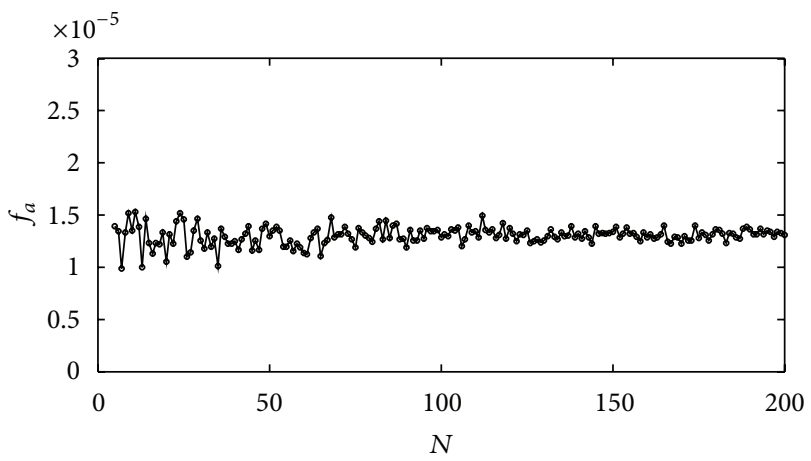

FIGURE 6: Relationship between $f_{a}$ and $N$ with intermittent release measures.

between adjacent fleets. Initially, the vehicles are randomly distributed within $2 \mathrm{~N}$ cells range of the starting point of the fog zone (as shown in Figure 5). Different values of $N$ are simulated, $N_{\min }=5$. The simulation was also repeated 20 times for getting the average value.

The space-time diagram of the fleet can reflect the characteristics of intermittent release measures on macro perspective. As seen in Figure 5, the length of the fleet gradually becomes longer and longer. And some drivers lose contact with the front vehicles and to be the laggers. The speed of the following vehicles slows down correspondingly. Therefore, the laggers become moving bottlenecks. However, the vehicle following distance may be shortened if the front vehicles slow down subsequently for some reason, and the laggers will see the front vehicles again, returning to following mode.

After intermittent release measures are taken, the value of $f_{a}$ is shown in Figure 6. Judging from order of magnitude, $f_{a}$ is about one-tenth of the original as shown in Figure 4. And the running time of the fleet passing through the fog zone has big change with $N$ increasing, as shown in Figure 7.

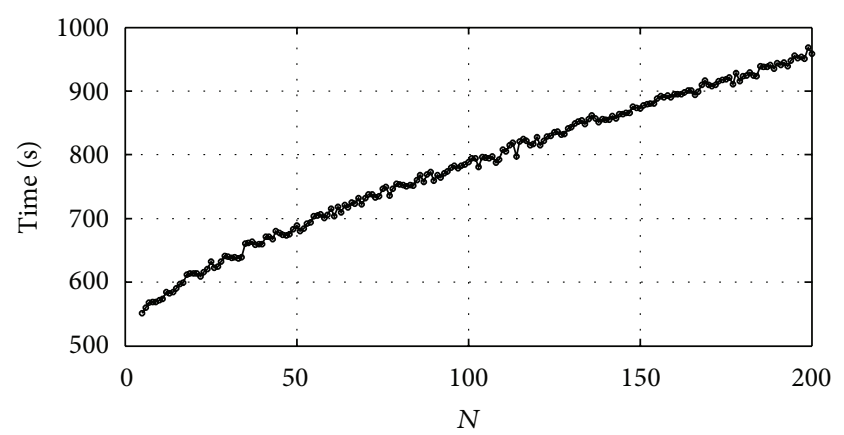

FIGURE 7: Relationship between running time and $N$.

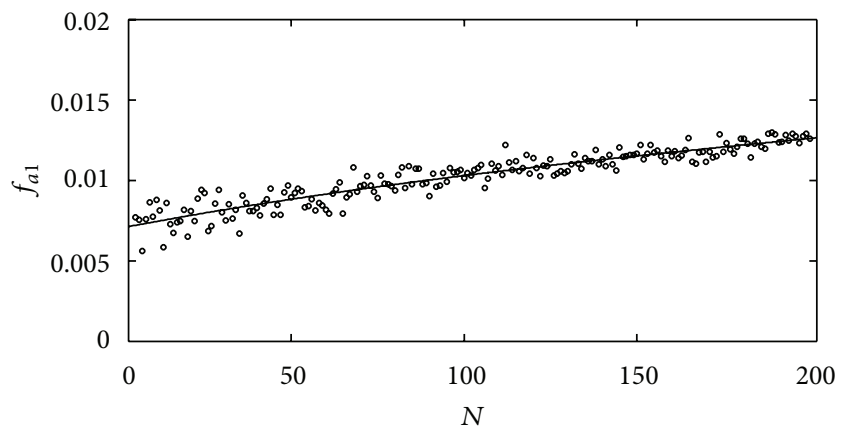

FIGURE 8: Relationship between $f_{a 1}$ and $N$.

Figure 8 shows the simulation results of the average probability of a car in a traffic accident when passing through the fog zone. The fitting curve equation $f_{a 1}$ is shown as (6)

$$
\begin{aligned}
f_{a 1}= & 1.371 \times 10^{-10} N^{3}-8.96 \times 10^{-8} N^{2} \\
& +4.039 \times 10^{-5} N+0.007005 .
\end{aligned}
$$

The time interval, represented by $h_{t}$, refers to the start time difference between adjacent fleets in passing through the fog zone. Figure 9 shows the simulation results of $h_{t}$, and the fitting curve equation is shown as

$$
\begin{aligned}
h_{t}= & -1.671 \times 10^{-7} N^{4}+9.697 \times 10^{-5} N^{3} \\
& -0.02122 N^{2}+4.85 N+12.4 .
\end{aligned}
$$

Furthermore, according to the time interval, we can use (8) to obtain the relationship between the maximum number of vehicles per hour $Q_{\max }$ and the number of each fleet. The simulation result is shown in Figure 10, and the fitting curve equation is shown as (9)

$$
\begin{gathered}
Q_{\max }=N \frac{3600}{h_{t}} . \\
Q_{\max }=-8.312 \times 10^{-7} N^{4}+0.0004269 N^{3} \\
-0.08586 N^{2}+9.644 N+534.2 .
\end{gathered}
$$




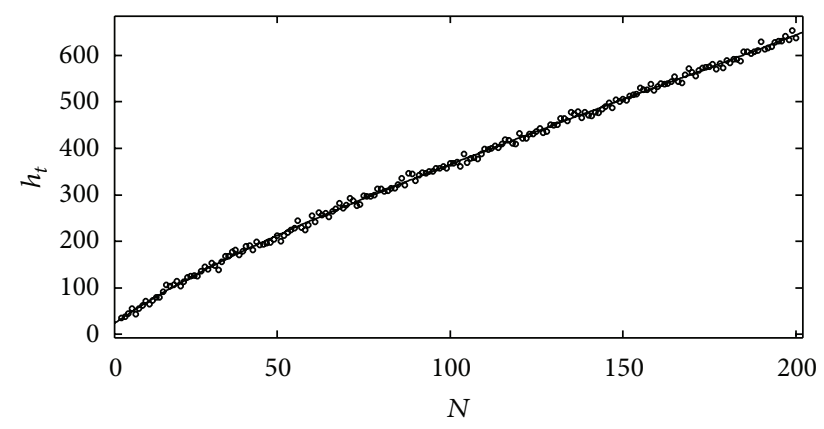

FIgURE 9: Relationship between $h_{t}$ and $N$.

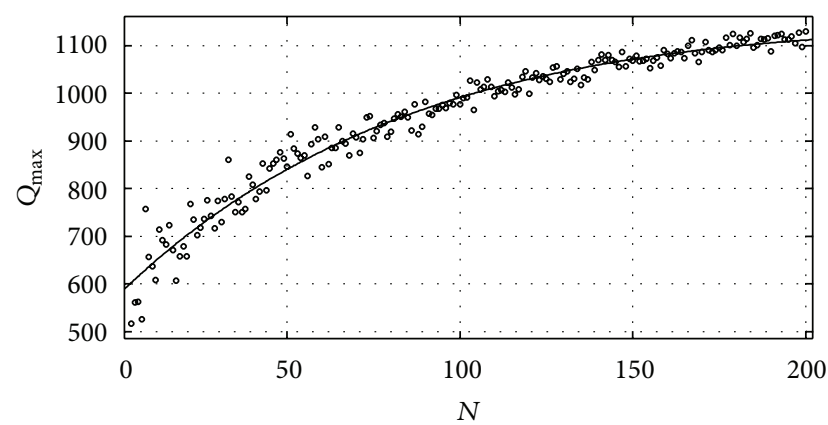

FIGURE 10: Relationship between $Q_{\max }$ and $N$.

\section{Conclusions}

To explore the impact of heavy fog on traffic, including speed, capacity, and safety, an improved cellular automaton model which considers specific driving behaviors was proposed in this paper. Meanwhile, the intermittent release measures were studied in order to keep freeway open to traffic and ensure traffic safety. The main conclusions are as follows.

(1) The heavy fog weather reduces traffic speed and capacity, and the probability of traffic accidents on average is much greater than normal weather when $\rho<0.5$.

(2) It shows that the proposed cellular automaton model of traffic can reflect the real driving behaviors and reproduce the situation, as Broughton et al. found that there were laggers in heavy fog weather. The simulation result of the probability of traffic accident shows that intermittent release measures can indeed reduce risk.

(3) When the fleet is passing through the fog zone, the more the vehicles $N$ are released, the higher the probability of traffic accidents is. Therefore, traffic management departments should select the appropriate release number of vehicles in practical applications. If $f_{a 1}<1 \%$ is assumed to be safe, from (6), (7), and (9), we know that the maximum vehicles $N$ in the fleet is 90 , the time interval between fleets is at least 337 seconds, and the $Q_{\max }$ is no more than 962 vehicles per hour. The results can provide a theoretical basis and reference for the traffic management department to make intermittent release measures.

\section{References}

[1] J. O. Brooks, M. C. Crisler, N. Klein et al., "Speed choice and driving performance in simulated foggy conditions," Accident Analysis and Prevention, vol. 43, no. 3, pp. 698-705, 2011.

[2] C. X. Zou, The distribution characteristics and risk assessment of fog disaster in Huning Highway [M.S. thesis], Nanjing University of Information Science \& Technology, Nanjing, China, 2011.

[3] Ministry of Public Security of the People's Republic of China, Announcement of the Freeway Traffic Management in Low Visibility Weather Conditions, Ministry of Public Security of the People's Republic of China, Beijing, China, 1997.

[4] K. L. M. Broughton, F. Switzer, and D. Scott, "Car following decisions under three visibility conditions and two speeds tested with a driving simulator," Accident Analysis and Prevention, vol. 39, no. 1, pp. 106-116, 2007.

[5] S. Caro, V. Cavallo, C. Marendaz, E. R. Boer, and F. Vienne, "Can headway reduction in fog be explained by impaired perception of relative motion?" Human Factors, vol. 51, no. 3, pp. 378-392, 2009.

[6] A. S. Mueller and L. M. Trick, "Driving in fog: the effects of driving experience and visibility on speed compensation and hazard avoidance," Accident Analysis and Prevention, vol. 48, pp. 472-479, 2012.

[7] J. Y. Duan, Driver's risk illusions in car following and unconcious imitation behavior [Ph.D. thesis], Tsinghua University, Beijing, China, 2012.

[8] M. Cremer and J. Ludwig, "A fast simulation model for traffic flow on the basis of boolean operations," Mathematics and Computers in Simulation, vol. 28, no. 4, pp. 297-303, 1986.

[9] K. Nagel and M. Schreckenberg, "A cellular automaton model for freeway traffic," Journal De Physique I, vol. 2, no. 12, pp. 22212229, 1992.

[10] M. Fukui and Y. Ishibashi, "Traffic Flow in 1D Cellular Automaton Model Including Cars Moving with High Speed," Journal of the Physical Society of Japan, vol. 65, no. 6, pp. 1868-1870, 1996.

[11] W. Knospe, L. Santen, A. Schadschneider, and M. Schreckenberg, "Towards a realistic microscopic description of highway traffic," Journal of Physics A, vol. 33, no. 48, pp. L477-L485, 2000.

[12] N. Boccara, H. Fukś, and Q. Zeng, "Car accidents and number of stopped cars due to road blockage on a one-lane highway," Journal of Physics A, vol. 30, no. 10, pp. 3329-3332, 1997.

[13] N. Moussa, "Car accidents in cellular automata models for onelane traffic flow," Physical Review E, vol. 68, no. 3, Article ID 036127, pp. 361271-361278, 2003.

[14] R. Jiang, X. Wang, and Q. Wu, "Dangerous situations within the framework of the Nagel-Schreckenberg model," Journal of Physics A, vol. 36, no. 17, pp. 4763-4769, 2003.

[15] M. E. Lárraga, J. A. del Río, and L. Alvarez-lcaza, "Cellular automata for one-lane traffic flow modeling," Transportation Research C, vol. 13, no. 1, pp. 63-74, 2005.

[16] K. F. Wu, L. J. Kong, and M. R. Liu, "The study of a cellular automaton NS and WWH mixed model for traffic flow on a twolane roadway," Acta Physica Sinica, vol. 55, no. 12, pp. 6275-6280, 2006.

[17] L. J. Peng and R. Kang, "One-dimensional cellular automaton model of traffic flow considering drivers' features," Acta Physica Sinica, vol. 58, no. 2, pp. 830-835, 2009. 
[18] J. X. Ding, H. J. Huang, and Q. Tian, "A traffic flow cellular automaton model to considering drivers' learning and forgetting behaviour," Chinese Physics B, vol. 20, no. 2, Article ID 028901, pp. 1-11, 2011.

[19] H. D. He, W. Z. Lu, and L. Y. Dong, "An improved cellular automaton model considering the effect of traffic lights and driving behaviour," Chinese Physics B, vol. 20, no. 4, Article ID 040514, pp. 1-7, 2011.

[20] Transportation Research Board, Highway Capacity Manual Fifth Edition (HCM, 2010), Transportation Research Board, Washington, DC, USA, 2010.

[21] L. Tao, Research on drivers' aberrant driving behavior styles and their discriminant indices [M.S. thesis], Tsinghua University, Beijing, China, 2012. 


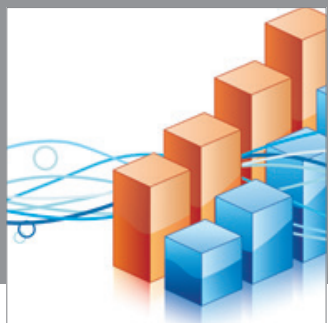

Advances in

Operations Research

mansans

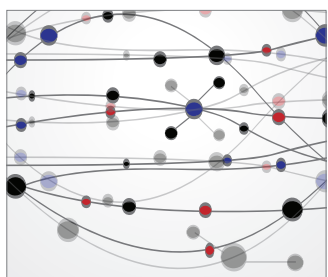

The Scientific World Journal
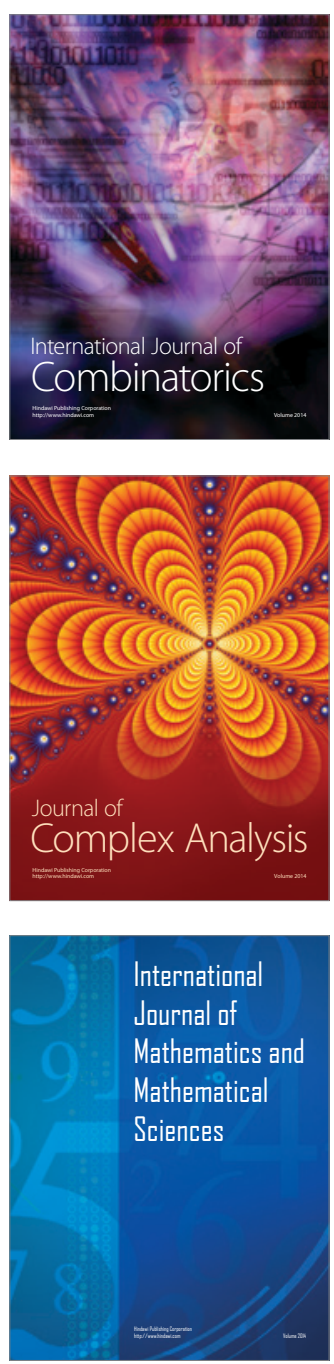
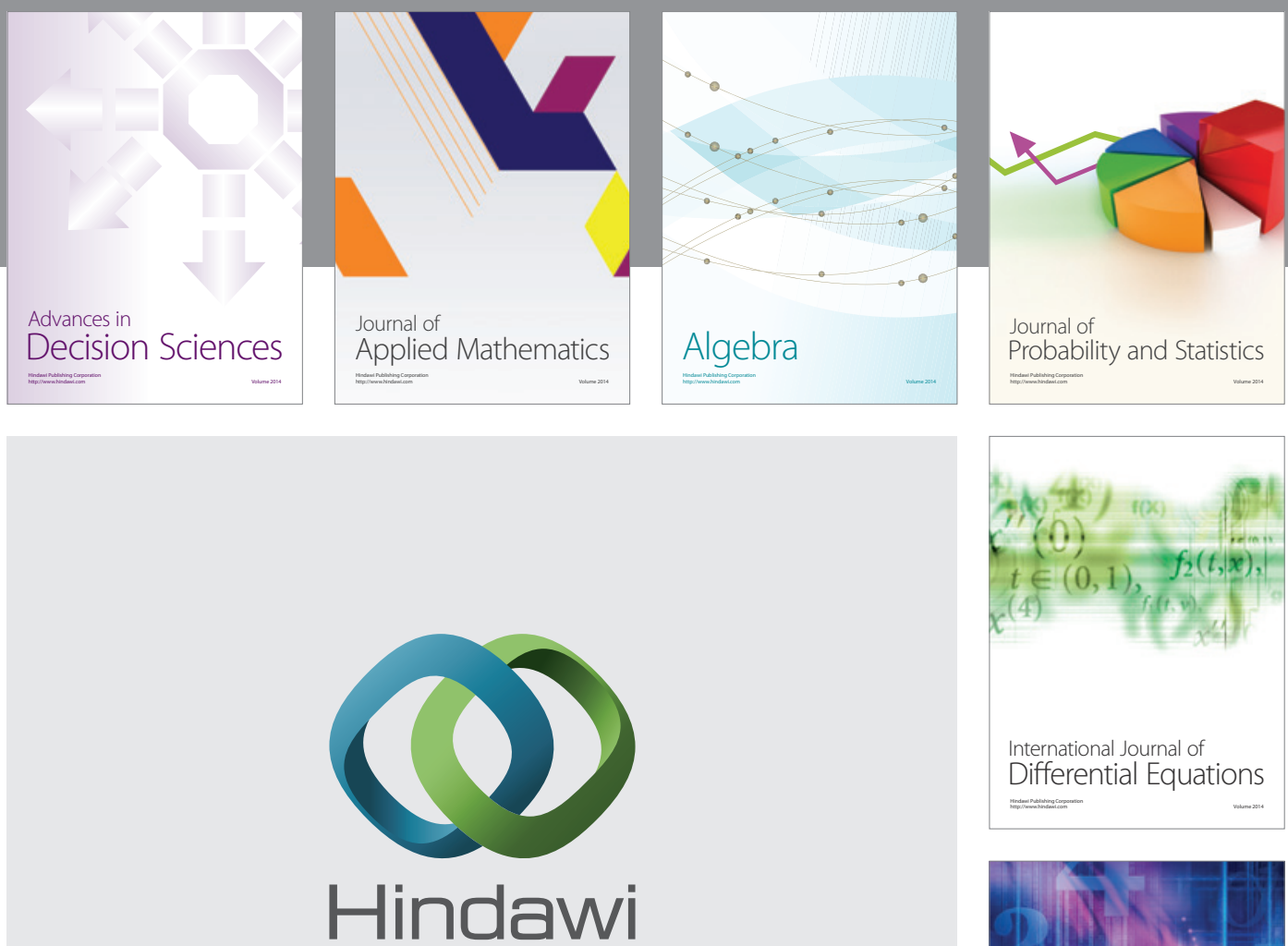

Submit your manuscripts at http://www.hindawi.com
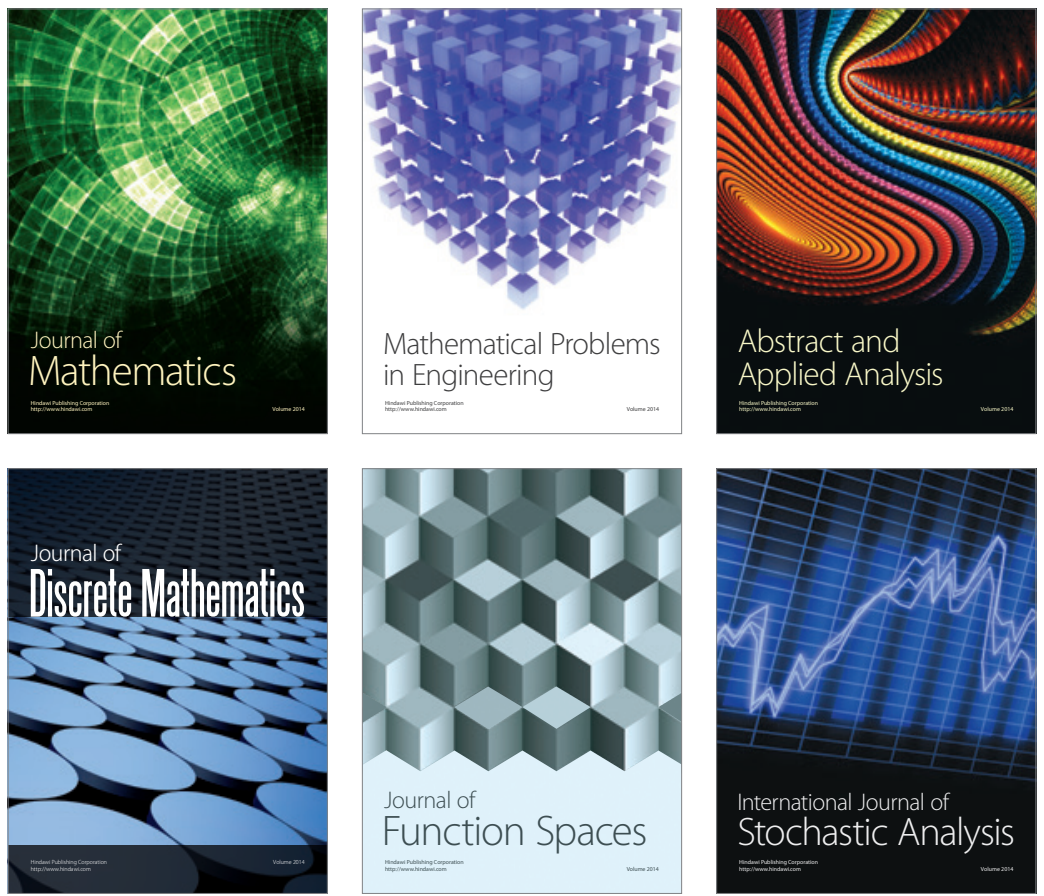

Journal of

Function Spaces

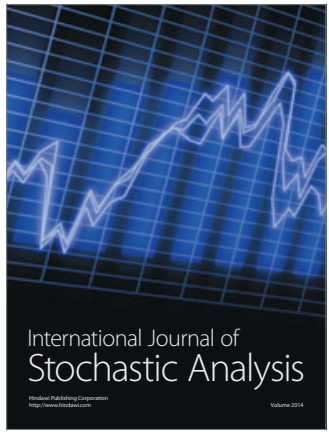

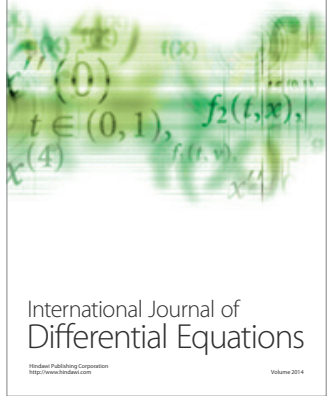
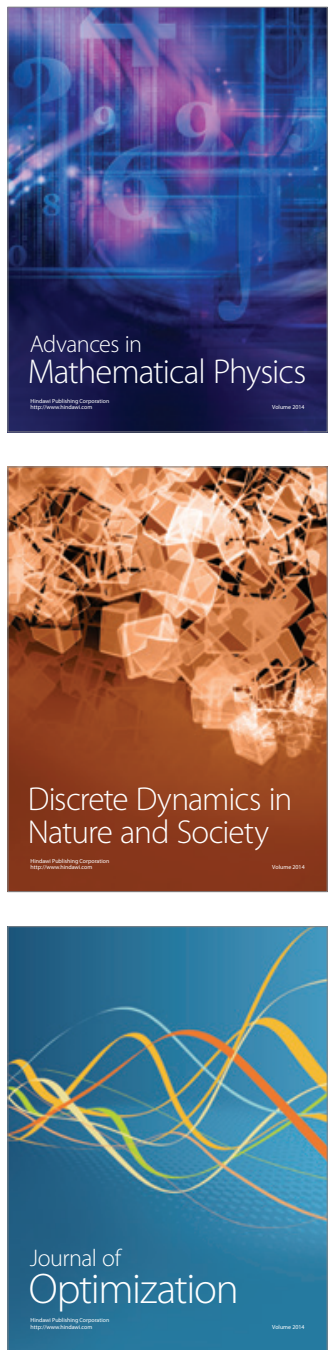